\title{
Looking for Emotions on a Single EEG Signal
}

\author{
Roylán Quesada-Tabares (D, Alberto J. Molina-Cantero ${ }^{(\otimes)}(\mathbb{D}$, \\ José I. Escudero-Fombuena(D), Manuel Merino-Monge(iD, \\ Isabel M. Gómez-González(D), Clara Lebrato-Vázquez(D), \\ and Juan A. Castro-García (iD
}

Departamento de Tecnología Electrónica, Universidad de Sevilla, Seville, Spain roylanqt@gmail.com, almolina@us.es

\begin{abstract}
This work aims at demonstrating that it is possible to detect emotions using a single EEG channel with an accuracy that is comparable to that obtained in studies carried out with devices that have a high number of channels. In this article the Neurosky Maindwave device, which only a single electrode at the FP1 position, the MatLab and the IBM SPSS Modeler were used to acquire, process and classify the signals respectively. It is remarkable the accuracy achieved in relation to the inexpensive hardware employed for the acquisition of the EEG signal. The result of this study allows us to determine when the brain response is more intense after undergoing the subject, in the experimentation, to the stimuli that generate those emotions. This let us decide which brain power bands are most significants and which moments are the most appropriate to carry out this detection of emotions.
\end{abstract}

Keywords: Emotions · Signal processing - Single EEG channel • Classification analysis $\cdot$ Dynamic properties

\section{Introduction}

Several theories have tried to explain the origin and nature of emotions. One of them posits the existence of a relative low number of basic emotions (families of emotions) which are universal for all human beings and independent of any cultural environment [8,12]. At least six emotional families have been proposed. They are: happiness, sadness, disgust, anger, fear and surprise. This theory includes, in turn, a locationist model that assumes that each emotional category starts from a specific place of the brain and body. Specifically, fear is located in the amygdala; disgust, in the insula; anger in the orbito-frontal cortex (OFC) and sadness in the anterior cortex of the cingulate (ACC) [28].

Another theory is based on the so-called constructionist model, in which it is asserted that emotions are psychological events that emerge from basic physiological operations and in which a large number of neural networks, spread in the 
brain, work together to obtain a meaningful experience. In [13] the authors propose a model with four components: core affect, some body sensory input that is experienced as pleasant/unpleasant with some degree of excitation; conceptualization, which links the body sensations with previous experiences to endow them with meaning; emotional words, used as support of emotional categories that are not clearly differentiable from the sensitive point of view; and executive attention, which focuses on some of the incoming stimuli. Some neuroimaging results have corroborated that, unlike that the locationist model predicts, a region activated during a basic emotion, was also for at least one other emotion [13]. This suggests the existence of neural networks that interact with each other to generate the emotions, instead of precise places (locationist model). For example the amygdala is recruited for both fear and disgust, so it takes different functionalities depending on the neural network that uses it.

There is no a dominant theory about another, taking into account the results of some neuroimaging studies, which can be interpreted differently according to the procedure used for the treatment of the data. [11] summarizes the existing controversy pointing out that the future should include analysis with animal models and studies of patients with brain injuries, which have been reported a tendency towards a locationist theory.

The dimensional theory considers that emotions can be represented in an Ndimensional space, where two of the coordinate axis would explain most of the emotional variations. These axis are called Valence and Arousal [19]. Valence is related to pleasure and varies from low values (very unpleasant) to high values (very pleasant). Arousal is related to the intensity of emotion, ranging from very low to very high. The dimensional model is also called the circumplex model, which is a dimensional representation of the core affect in the constructionist model. Although it would seem more appropriate to use this representation for the constructionist model, in fact, the six basic emotions can also be characterized according to their valence and arousal.

Emotions can be detected in several ways: through the analysis of gestures (facial), speech [16] or the activity of various physiological signals, such as the electroencephalogram (EEG). In this work we analyze the EEG to obtain the activity of the brain during experiment in which specific emotions are elicited.

There are a large number of commercial devices for measuring and recording the EEG activity. They vary in price and the way in transmitting the information (wired or wireless). We opted for the use of a wireless device, for its advantages in mobility. Several devices with these characteristics can be found in the market: Emotiv [10], Neurosky Mindwave [10] and Enobio [9]. Emotiv offers better results than the Neurosky Mindwave when it is used for the evaluation of cognitive processes [7]. However, the Neurosky Mindwave usability and prices make it very competitive. This device has been widely used by the scientific community for the development of various applications such as the detection of sleepiness [27], level of attention [14], stress [6,17], and so on. It is a device that offers developers and researchers the possibility to make a treatment of the measured signal, but also comes integrated with a system that processes and delivers to the user characteristics of the post-processing, which will not be used in our case. 
For the study and comprehension of the EEG signal, the analysis of the bands is widely used [20]. They are: $\delta$ (which ranges from 0 up to $4 \mathrm{~Hz}), \theta(4-8 \mathrm{~Hz}), \alpha$ $(8-12 \mathrm{~Hz}), \beta(13-30 \mathrm{~Hz})$ and $\gamma(>30 \mathrm{~Hz})$. Another feature we have used to study the EEG signal is the fractal dimension $[22,29]$. The fractal dimension shows the complexity of the signal. There are several ways to calculate it, in this study we used the Higuchi's algorithm [26]. A fractal dimension close to 2 indicates that the signal is very complex, however a value close to 1 means that the signal is close to a line.

This study aims to keep on analyzing the features extracted from a single EEG signal, which started in our previous work [18], but adding the statistical significance when different temporal windows of data are used to make the classification of the emotional states elicited by sets of images.

\section{Methodology}

\subsection{Materials}

The EEG signal was captured using the Neurosky Mindwave, which has a fixed electrode located at Fp1, uses a sampling frequency at $512 \mathrm{~Hz}$, a 12 bits analogdigital converter and a bluetooth interface to transmit the raw signal. To read, save, show the pictures and process data we used Matlab 8.4.0.150421 (R2014b). Finally, the IBM SPSS Modeler, a software with data mining tools, was employed to analyzed and classify the features extracted from raw EEG signals. IBM SPSS Modeler offers a wide variety of modeling methods from automated learning, artificial intelligence and statistics [5].

\subsection{Experimentation}

The experimentation consisted in showing 60 images (Table 1) selected from the IAPS (International Affective Picture System), grouped in three different sets of valence and arousal pairs (Fig.1). An overall of three different arousal and valence values were chosen: $2.306 \pm 0.43,5.063 \pm 0.24$ and $6,921 \pm 0.032$ for valence and sets 1, 2 and 3 respectively, whereas for arousal, $6.1890 \pm 0.04,3,020$ \pm 0.02 and $4,551 \pm 0.02$ for respective sets.

Seven people took part in the experiment with a mean age of 29.85 and a standard deviation of 8.97. Before starting the experiment, the SAM test (Self-assessment manikin) was applied to each person to evaluate her/his initial valence and arousal at that moment.

Each picture was displayed on a computer screen for six seconds (Fig. 2) following the same experiment schedule performed in [1] with a resting period of time among images of $4 \mathrm{~s}$, in which a cross was shown on the screen. A Matlab software application was responsible in displaying the images, registering the temporary marks to identify the time when the pictures appeared and were removed from the screen, and in receiving the raw EEG data sent by the Neurosky. All the information was stored for further processing. 
Table 1. Selected pictures obtained from the IAPS database. The arousal and valance for each picture and its averages are also shown.

\begin{tabular}{l|l|l|l|l|l|l|l|l}
\hline Set 1 & \multicolumn{5}{l}{ Set 2} & \multicolumn{3}{l}{ Set 3 } \\
\hline File & Valence & Arousal & File & Valence & Arousal & File & Valence & Arousal \\
\hline 2352.2 & 2.090 & 6.250 & 2038 & 5.090 & 2.940 & 1540 & 7.150 & 4.540 \\
\hline 2683 & 2.620 & 6.210 & 2102 & 5.160 & 3.030 & 1731 & 7.070 & 4.560 \\
\hline 3103 & 2.070 & 6.060 & 2381 & 5.250 & 3.040 & 2030 & 6.710 & 4.540 \\
\hline 3150 & 2.260 & 6.550 & 2411 & 5.070 & 2.860 & 2152 & 6.930 & 4.500 \\
\hline 3195 & 2.060 & 6.360 & 2850 & 5.220 & 3 & 2153 & 6.980 & 4.400 \\
\hline 3550.1 & 2.350 & 6.290 & 2880 & 5.180 & 2.960 & 2306 & 7.080 & 4.460 \\
\hline 6021 & 2.210 & 6.060 & 2890 & 4.950 & 2.950 & 2344 & 6.720 & 4.710 \\
\hline 6022 & 2.140 & 6.090 & 6150 & 5.080 & 3.220 & 2362 & 6.740 & 4.600 \\
\hline 6200 & 2.710 & 6.210 & 7003 & 4.970 & 3.160 & 2373 & 6.970 & 4.500 \\
\hline 6244 & 2.190 & 6.010 & 7004 & 5 & 3.070 & 2391 & 7.110 & 4.630 \\
\hline 6350 & 2.310 & 6.990 & 7010 & 4.930 & 3.010 & 2655 & 6.880 & 4.570 \\
\hline 6370 & 2.230 & 6.330 & 7018 & 5.180 & 3.120 & 5199 & 6.930 & 4.700 \\
\hline 6570.1 & 2.190 & 6.240 & 7035 & 4.950 & 3.060 & 5300 & 6.910 & 4.360 \\
\hline 6825 & 2.380 & 6.290 & 7054 & 5.220 & 2.950 & 5890 & 6.670 & 4.600 \\
\hline 9301 & 2.260 & 6 & 7056 & 4.900 & 3.020 & 5994 & 6.800 & 4.610 \\
\hline 9330 & 2.210 & 5.890 & 7057 & 5.070 & 3.070 & 7400 & 6.840 & 4.560 \\
\hline 9610 & 2.480 & 6.460 & 7161 & 5.020 & 3.070 & 7430 & 6.910 & 4.550 \\
\hline 9621 & 2.700 & 6.110 & 7165 & 4.980 & 2.980 & 7472 & 7.080 & 4.640 \\
\hline 9903 & 2.330 & 6 & 7180 & 5.060 & 2.880 & 7481 & 7.080 & 4.550 \\
\hline
\end{tabular}

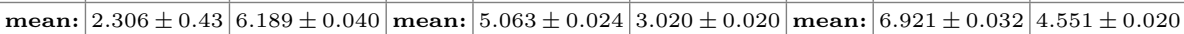

A webcam recorded the experiment in order to contrast any possible anomaly in the signal. Finally, people were asked to fill in the SAM test for the 60 images. To do this, they were shown the same pictures again, in the same order.

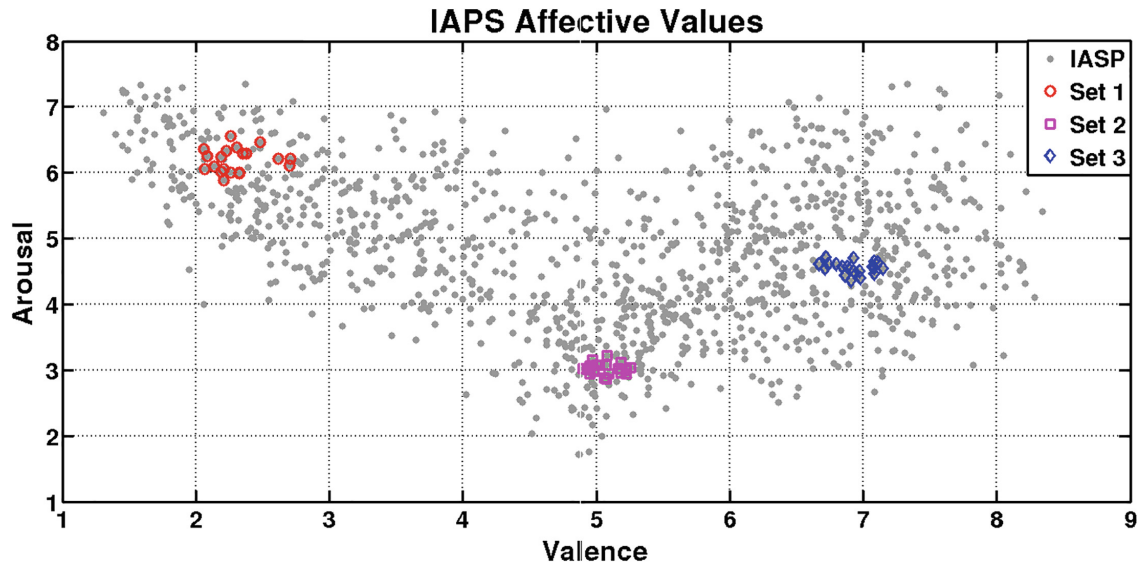

Fig. 1. Valence and arousal values of the selected pictures. Source [18]. 


\subsection{Signal Processing}

The EEG signal was split in epochs of 512 samples with an overlapping of $87.5 \%$ (or hop size of 64 samples). Then, each epoch was automatically analyzed to know if it was contaminated with artifacts: blinks, eye movements, muscle activity (EMG) or motion artifacts. In this specific case, the main source of artifacts are blinks and ocular movements, due to the electrode position, very close to the eye, although the electrical activity of the frontal an temporal muscles and motion artifacts are also important.

The automatic identification of contaminated epochs is based on obtaining two features: the difference between the maximum and minimum value (MinMax) in the epoch and the total energy of the signal (ESF) after applying a Savitzky-Golay lowpass filter (order 2 and length 35) [21]. Figure 3 shows the feature space. Epochs contaminated by muscular activity have values of the MinMax feature similar or a bit higher to those of the epochs with only EEG, but with more energy from the filtered signal (ESF). Blinking or EEG-only epochs have similar values in the ESF feature but differ from MinMax. Epochs with motion artifact contain values of these features that surround those obtained by other types of artifacts. An artificial neural network (ANN) was trained to detect epochs containing valid EEG data or blinks, but the results were similar to those obtained by setting thresholds in each dimension of the feature space (as shown in Fig. 3). Namely, the accuracy in detecting epochs contaminated with blinks was $98 \%$ whereas the accuracy in detecting valid EEG was of $96 \%$. Setting thresholds in the feature space makes the identification method of valid epochs to be conservative, reducing the number of false positives at the cost of increasing the number of false negatives. This means that is better to reject some valid epochs than to accept contaminated epochs.

While motion and EMG artifact are infrequent, the ocular ones do not. It is known that ocular artifacts affect, mainly, the lowest energy bands $(\delta, \theta$ and part of $\alpha$ ), so not to remove them from the epoch (or not to discard the epoch) could distort the frequency analysis. There are techniques for removing ocular artifacts from the EEG signal. One of the most well-known technique is based on the analysis of independent components (ICA) [24], but, for its application, at least two EEG channels are needed, which do not exist in our case. [25] shows a technique to eliminate such artifacts in a single channel. It is based on applying a Savitzky-Golay low pass filter with the same characteristics as the one used for the calculation of the ESF feature. Therefore, if an epoch is identified as a blinking container, such a filter is applied before proceeding to the frequency analysis.

Epochs containing valid EEG data or blinks, which were previously removed, were then windowed with a Hamming function to reduce spectral leakage. Then the squared fast Fourier transform (FFT) was applied to each segment to obtained the typical energy bands: $\delta, \theta, \alpha, \beta$ and $\gamma$. The fractal dimension was also calculated based on Higuchi's algorithm [15], so a total of 6 values per epoch were obtained. 


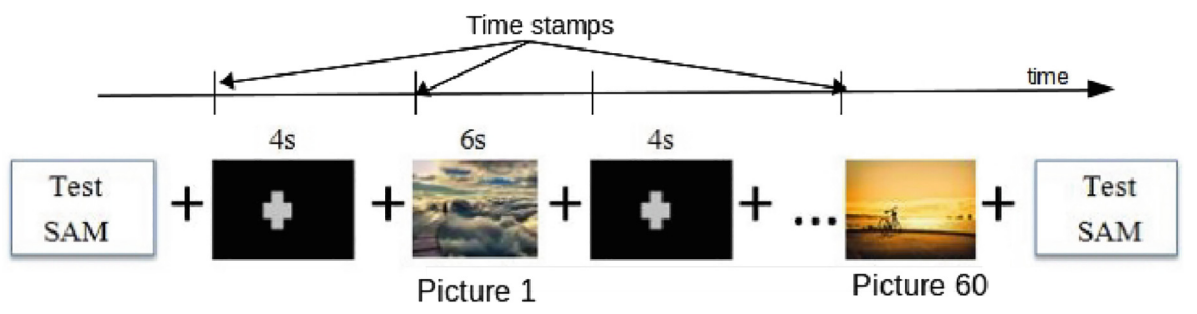

Fig. 2. Experimental sequence. Source [18].

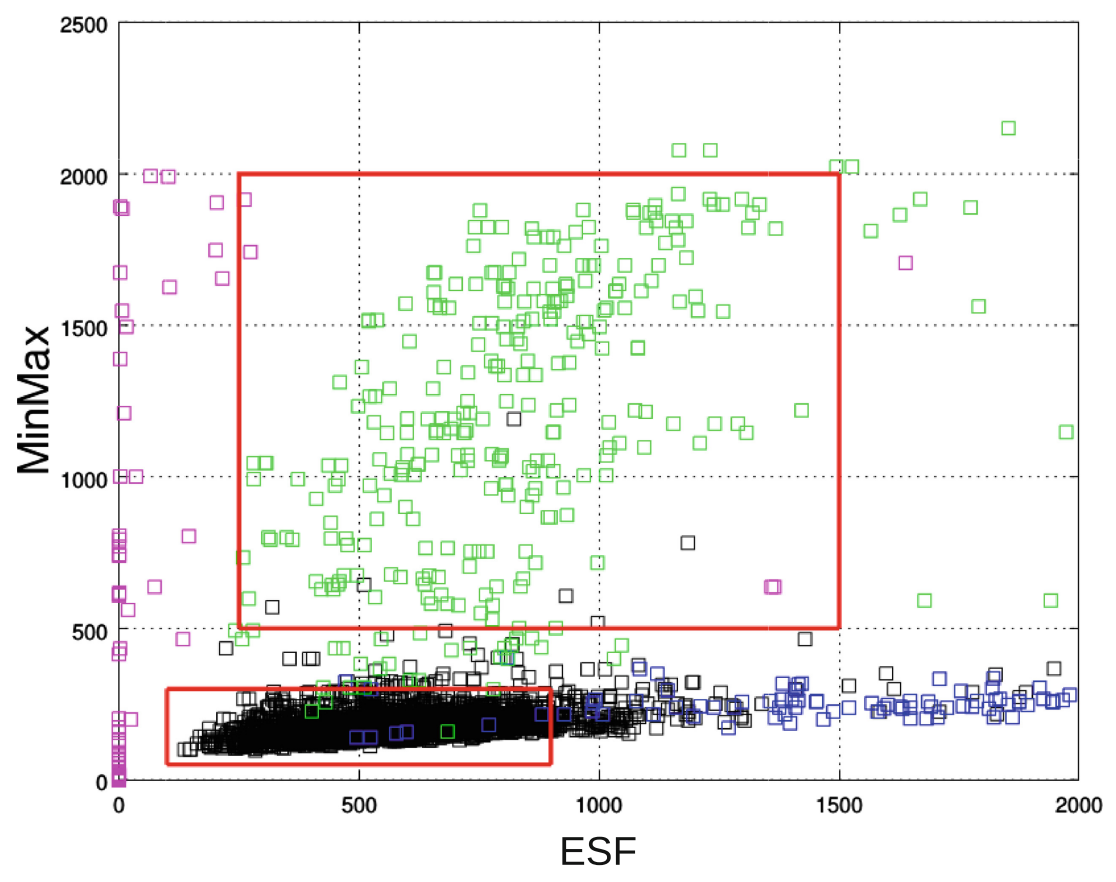

Fig. 3. Feature spaces values for motion artifacts (pink), muscular activity (blue), blinks (green) and EEG (black). The selected areas to identify EEG and EEG+blinks epochs are also shown. Source [18] (Color figure online)

\subsection{Analysis}

For each subject, the average of five typical power bands, the fractal dimension and some ratios such as $\theta / \beta$ and $\theta / \alpha$ for all valid epochs were obtained. This yields an overall of $60 \times 8$ numerical values for each subject, grouped into three sets, according to the valence/aerosal values shown in Table 1, or, in other words, $(3 \times 20 \times 8)$ features. Next, we applied three different types of analysis: statistical, classification and temporal. The first seeks to find significant differences among features and sets. The second analysis seeks to obtain the accuracy 
in detecting one out of the three emotional sets through a classifier based on decision trees. The last one studies the dynamic in inducing an emotion.

Study 1. Here, we looked for the statistical significance of some features among sets. For doing so, we employed the Kruskal-Wallis test, which is a nonparametric alternative to the analysis of variance (ANOVA). It serves to contrast the hypothesis that $\mathrm{k}$ quantitative samples haven been obtained from the same population. It requires samples (or features from pictures) to be obtained randomly, which is completely fulfilled by the experiment design. The statistical analysis is individually applied to each participant and feature, so $8 \times 7$ (features $\times$ subjects) analysis were carried out. Remind that for each picture there are 8 features estimated using a six-second segment of data.

Study 2. The IBM SPSS Modeler software was used in this analysis to generate a decision tree with the C5.0 classification algorithm. The option to partition the data, to ensure that the information used to test the model is not the same as the one employed to generate it, was activated. The input parameters to the classifier are the $8 \times 20 \times 3$ (features $\times$ pictures $\times$ sets). The outputs are the predicted sets. The classifier was trained for each person, so 7 training and testing processes were accomplished.

Study 3. Features in study 1 were obtained by using the whole 6 s segment of data associated to the visualization of each picture. Our hypothesis is that features are not steady during the whole segment, but they show a temporal evolution. This is based on the idea that any subject takes a while to respond to the emotion elicited by the picture. As can be seen in Fig. 4, initially it must be a delay time (D), after the onset of the picture, in which the subject's responsiveness is low and start growing up. During the interval $\Delta T$, the feature reaches its maximum (minimum) value and probably began declining. It might be better to use only the interval $\Delta T$ instead of the whole segment for testing the statistical significance.

\section{Results}

\subsection{Study 1}

Table 2 shows the absolute values of the features obtained for Study 1, grouped according to the set of images and subject. Statistically significant values, with a $p<0.05$ have been also highlighted. Subjects 3, 6 and 7 obtained values significant for the fractal dimension, subjects 5 and 7 in the $\alpha$ band and subject 5 in the $\gamma$ power band too. There isn't a clear relationship between features' behavior and changes on valence and arousal. For example, for subject 3, the fractal dimension has a concave shape when moving from set 1 to set 3 whereas for the subject 6 , the same feature, has a growing monotone behavior. 


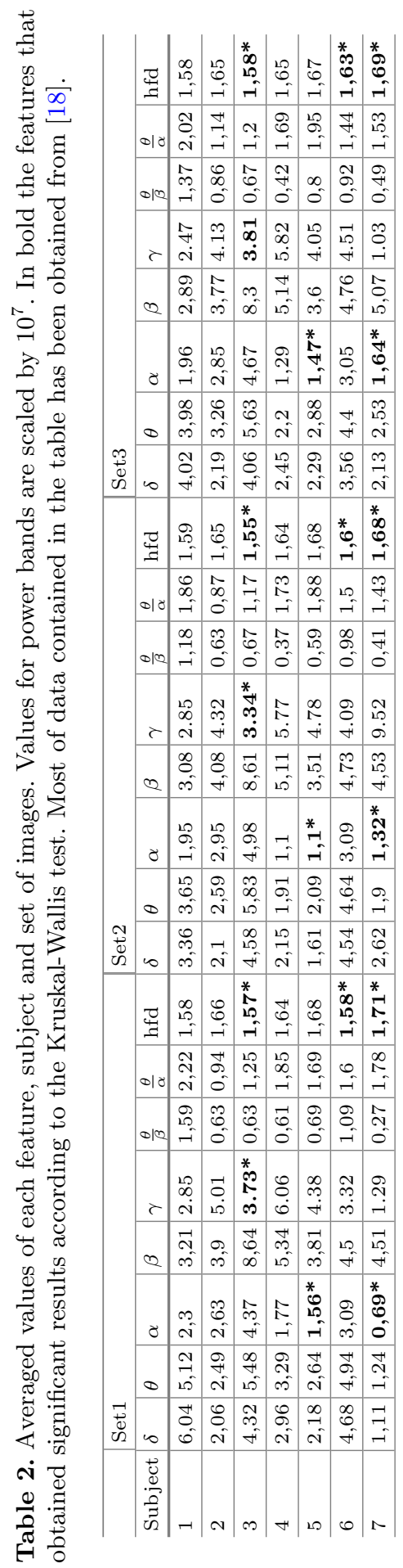


Subjects 5 and 7 show significant changes in the $\alpha$ band. However, like with the fractal dimension, there isn't a regular pattern in this feature. For example, while subject 7 shows an increase in $\alpha$, subject 5 , has a concave behavior.

Subject 3 also obtained significant values in $\gamma$ band with a concave behavior among sets.

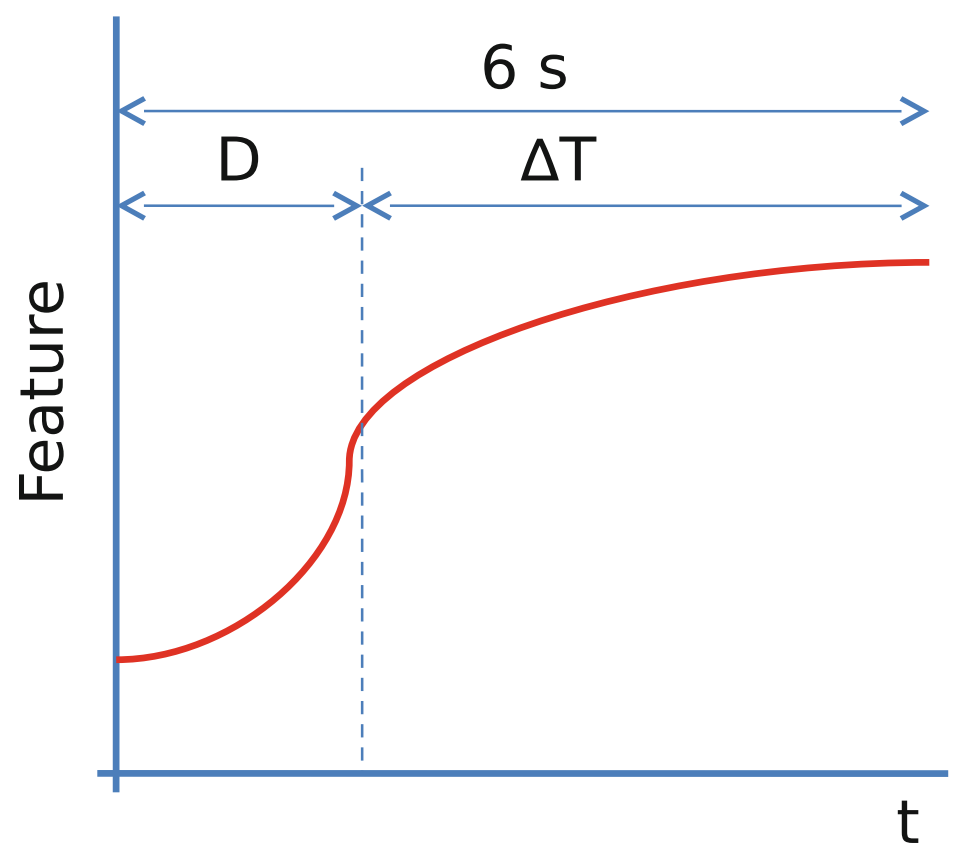

Fig. 4. Temporal evolution of a feature after the onset of the stimulus $(t=0)$. There is an initial delay, $\mathrm{D}$, followed by the interval of interest, $\Delta T$, in which the feature reaches its maximum or minimum value.

\subsection{Study 2}

Table 3 shows the accuracy in classifying the 3 sets. For them all, the accuracy is over $70 \%$, reaching up to near $92 \%$ for subject 5 . In average, the accuracy was around $81 \%$.

\subsection{Study 3}

We hypothesized that there must be a temporal evolution in any feature after the onset of the stimulus. To illustrate this Fig. 5 shows the average temporal evolution of $\alpha$ and $\gamma$ power bands for subject 4 and set of pictures. It can be seen that power bands temporally changes as time goes by. For example, in set 3 , it would be better to obtain this features in the middle of the interval for the $\alpha$ band. 
Table 3. Accuracy in detecting emotional states.

\begin{tabular}{l|l}
\hline Subject & Accuracy \\
\hline 1 & $78,33 \%$ \\
\hline 2 & $78,33 \%$ \\
\hline 3 & $76,67 \%$ \\
\hline 4 & $73,33 \%$ \\
\hline 5 & $91,67 \%$ \\
\hline 6 & $81.67 \%$ \\
\hline 7 & $85 \%$ \\
\hline Mean & $\mathbf{8 0 . 7 1 \%}$ \\
\hline
\end{tabular}
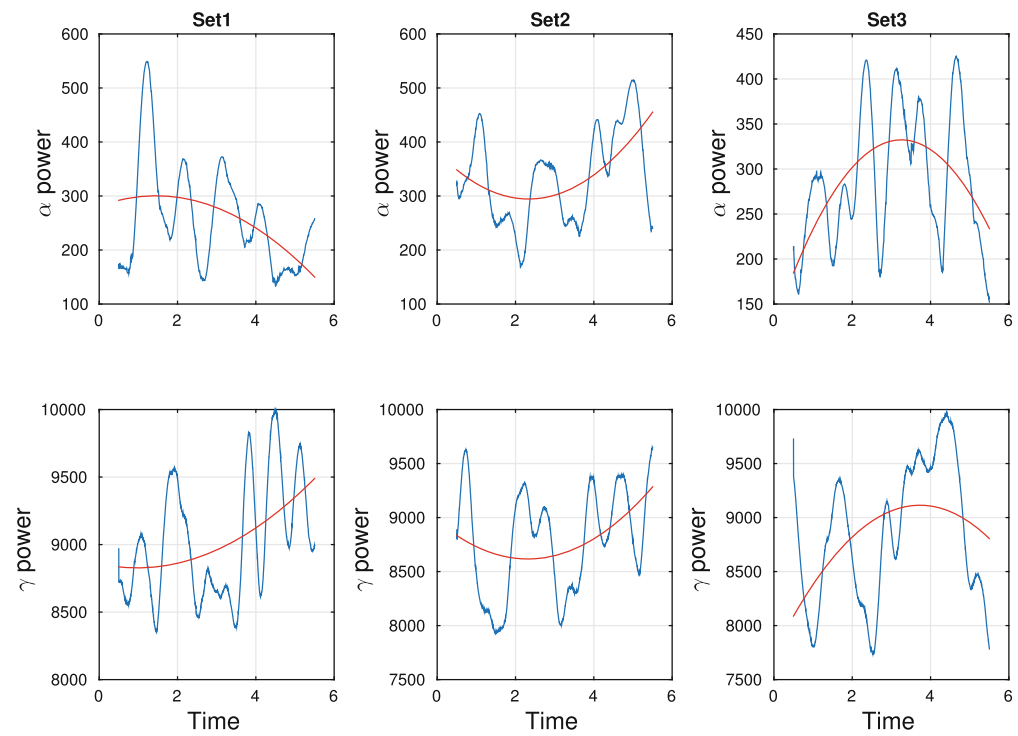

Fig. 5. Temporal evolution of $\alpha$ and $\gamma$ power bands for participant 4 using 1s-length analysis windows. A parabolic interpolation curve is also shown.

Table 4 shows, for the different possible values of $\mathrm{D}$ and $\Delta T$, how many statistically significant values $(p<0.05)$ are given in the different features under study $(\delta, \theta, \alpha, \beta, \gamma$ and hfd). The first thing to keep in mind is that in these tables the values below the diagonal do not make sense, since always $D+\Delta T$ must be less than or equal to $6 \mathrm{~s}$. The most interesting results are obtained in the $\alpha$ and $\gamma$ bands and hfd, andto a lesser extent in $\theta$ band. As seen, the $\beta$ and $\delta$ bands are the least statistical relevance. It is also easily seen, that the best pair of values for $(\mathrm{D}, \Delta T)$ are: $(4,2) ;(3,3) ;(2,4)$ and to a lesser extent $(1,5)$. 
Table 4. Number of subjects with significant features according to the statistical analysis, the interval $\Delta T$ and the delay D.

a) $\delta$ power band

\begin{tabular}{|c|c|c|c|c|c|c|}
\hline & \multicolumn{6}{|c|}{$\Delta T(\mathrm{~s})}$. \\
\hline $\mathrm{D}(\mathrm{s})$. & 1 & 2 & 3 & 4 & 5 & 6 \\
\hline 0 & 0 & 0 & 0 & 0 & 0 & 1 \\
\hline 1 & 0 & 0 & 0 & 0 & 1 & \\
\hline 2 & 0 & 2 & 1 & 1 & & \\
\hline 3 & 0 & 0 & 0 & & & \\
\hline 4 & 0 & 1 & & & & \\
\hline 5 & 1 & & & & & \\
\hline
\end{tabular}

c) $\alpha$ power band b) $\theta$ power band

\begin{tabular}{|c|c|c|c|c|c|c|}
\hline & \multicolumn{6}{|c|}{$\Delta T$ (s.) } \\
\hline $\mathrm{D}(\mathrm{s})$. & 1 & 2 & 3 & 4 & 5 & 6 \\
\hline 0 & 0 & 0 & 0 & 0 & 0 & 0 \\
\hline 1 & 0 & 0 & 1 & 0 & 1 & \\
\hline 2 & 1 & 2 & 1 & 2 & & \\
\hline 3 & 0 & 0 & 2 & & & \\
\hline 4 & 0 & 2 & & & & \\
\hline 5 & 2 & & & & & \\
\hline
\end{tabular}

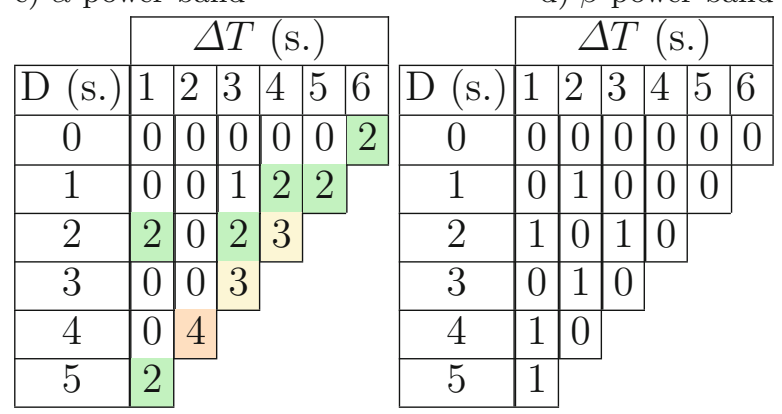

e) $\gamma$ power band

f) Fractal dimension

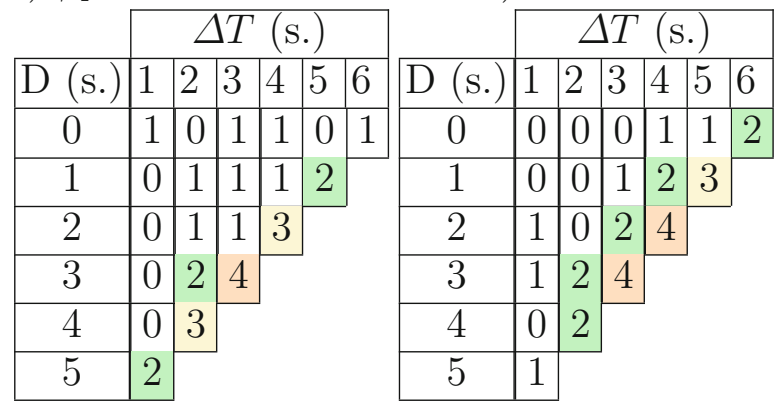

\section{Discussion}

The fractal dimension is related to the complexity of the EEG signal, which is increased in neural activation processes. In our previous work [18], it was suspected the existence of a relationship between arousal and fractal dimension. In this new study we have included the $\gamma$ power band which, in turn, can be also considered as an indicator of underlying neural activation. Only one subject 
obtained significant differences in this feature among sets, with a behavior that suggests a correlation with the arousal dimension. With respect to valence, [18] showed that it is very difficult to establish any kind of correlation with only one electrode since it does not capture lateralization effects. These effects between hemispheres have been found in some studies [1]. For negative valences it was observed a greater synchronization (activation) in the left hemisphere, whereas for positive valences, the same effect was observed in the opposite hemisphere.

The averaged accuracy in detecting these three emotional sets was approximately of $81 \%$ when using a decision tree as classifier. Table 5 summarizes a comparative between our results and other studies, highlighting the number of electrodes used and the emotions detected. As can be seen, our results outperforms previous work regarding the same number of electrodes [30] although the number of emotional states was slightly higher in such a study. Regarding to the same number of emotions to be detect, in this study we almost equal other works with higher number of electrodes [3]. For a more detailed discussion in this issue see [18].

Table 5. Accuracy in detecting emotional states.

\begin{tabular}{l|l|l|l}
\hline Author & $\begin{array}{l}\text { Number of } \\
\text { electrodes }\end{array}$ & $\begin{array}{l}\text { Number of } \\
\text { emotions }\end{array}$ & Accuracy \\
\hline H. Yoon [30] & 1 & 4 & $66 \%$ \\
\hline L. Brown [3] & 9 & 3 & $82 \%$ \\
\hline O. Sourina [23] & 3 & 4 & from 70\% up to 100\% \\
\hline D. O. Bos [2] & 3 & 5 & $\begin{array}{l}92,3 \% \text { (valence) } \\
97,4 \% \text { (arousal) }\end{array}$ \\
\hline G. Chanel [4] & 64 & 3 & $\begin{array}{l}76 \% \text { (valence) } \\
67 \% \text { (arousal) }\end{array}$ \\
\hline R. Quesada [18] & 1 & & $80.71 \%$ \\
\hline
\end{tabular}

Data support the dynamic in the elicitation of an emotion. There exists an initial delay, which can vary between $1-2 \mathrm{~s}$, wherein the physiological processes triggered by the picture visualization are emerging. When considering the whole interval of picture exposition, the estimated features may be pulled down by this initial phenomena. So, it is worthy to consider the assessment of the feature several seconds after the onset of the stimulus in order to give time to the internal process to get to a steady state.

An explanation of why there were only a few significant features can be seen in Fig. 6. It shows the averages of reported arousal and valence for each set of pictures per subject (circle) and the averages for all subjects $\left(^{*}\right)$.

Although it can be seen that averages per subject approximate to the values of the IAPS (except for set1, whose valence is greater and the arousal is lower), the dispersion of the values reported for each of the 20 pictures is quite higher 
than those indicated in the IAPS database. This could justify, in large part, why it has not been obtained significant variations among the measured features for the different sets of pictures.

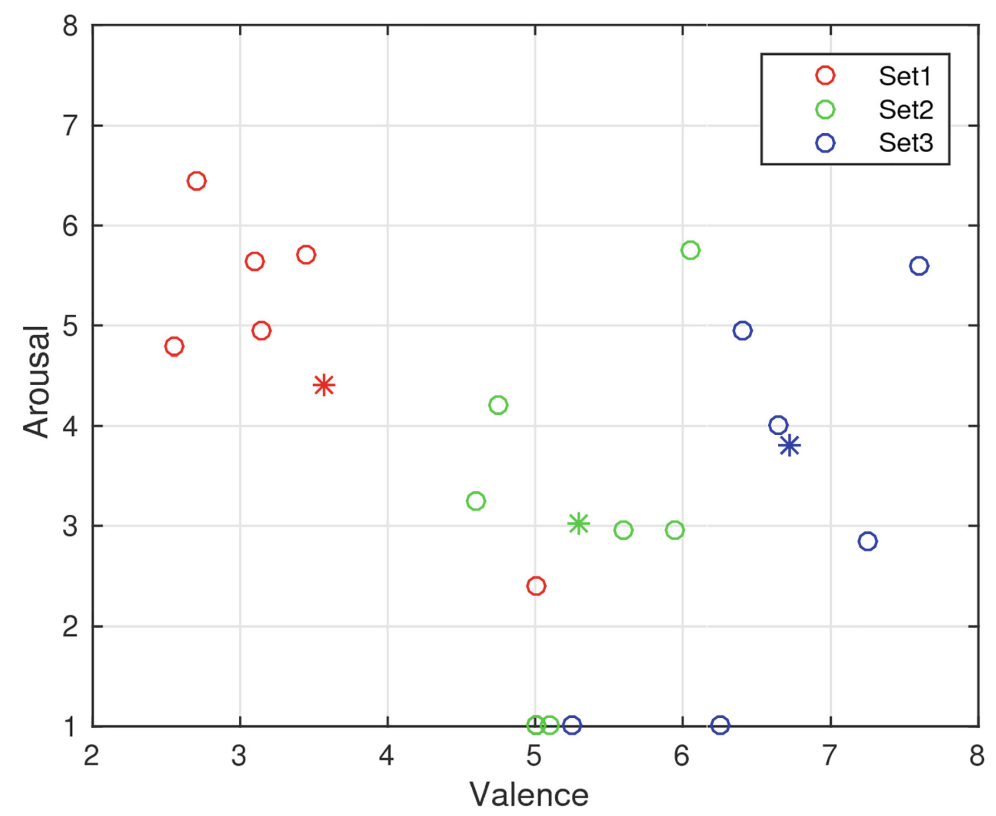

Fig. 6. Mean experimental valence and arousal values for each set of pictures and subject. Source [18].

\section{Conclusions and Future Work}

This study has shown the feasibility of using a device, with a single EEG at the frontopolar position, for registering the significant neural activity during experiments involving the elicitation of a small set of emotions. The detection accuracy was over $80 \%$, which outperforms some results reported in the scientific literature. The most significant features were the $\alpha$ and $\gamma$ power bands and the fractal dimension, hfd. It has also been proved that there is an improvement in the significance of results when the dynamic effect in inducing an emotion is considered. Namely, the number of subjects that obtained significant values in such bands increased when the delay $\mathrm{D}$ was higher than $2 \mathrm{~s}$ and the interval of analysis $\Delta T$ reduced to $2-4 \mathrm{~s}$.

It is of interest for subsequent studies to make a similar investigation taking into account the following considerations: that the set of pictures vary in a single dimension of the circumplex model when passing from one set to another; increase considerably the number of people performing the experimental test; 
use the incremental feature variation between the the analysis window and the preceding resting period; and finally take into account the values of $\mathrm{D}$ and $\Delta T$ to improve the classification results.

Acknowledgments. We sincerely and deeply thank the people involved in the realization of this study and the anonymous reviewers who helped us improve this document with their comments.

\section{References}

1. Aftanas, L., Varlamov, A., Pavlov, S., Makhnev, V., Reva, N.: Affective picture processing: event-related synchronization within individually defined human theta band is modulated by valence dimension. Neurosci. Lett. 303(2), 115-118 (2001)

2. Bos, D.O., et al.: EEG-based emotion recognition. The Influence of Visual and Auditory Stimuli, pp. 1-17 (2006)

3. Brown, L., Grundlehner, B., Penders, J.: Towards wireless emotional valence detection from EEG. In: 2011 Annual International Conference of the IEEE Engineering in Medicine and Biology Society. pp. 2188-2191. IEEE (2011)

4. Chanel, G., Ansari-Asl, K., Pun, T.: Valence-arousal evaluation using physiological signals in an emotion recall paradigm. In: 2007 IEEE International Conference on Systems, Man and Cybernetics, pp. 2662-2667. IEEE (2007)

5. Corporation, I.: Manual de usuario de IBM SPSS Modeler 15, p. 280 (2012). ftp://public.dhe.ibm.com/software/analytics/spss/documentation/modeler/15.0/ es/UsersGuide.pdf

6. Crowley, K., Sliney, A., Pitt, I., Murphy, D.: Evaluating a brain-computer interface to categorise human emotional response. In: 2010 10th IEEE International Conference on Advanced Learning Technologies. pp. 276-278. IEEE (2010)

7. Das, R., Chatterjee, D., Das, D., Sinharay, A., Sinha, A.: Cognitive load measurement-a methodology to compare low cost commercial EEG devices. In: 2014 International Conference on Advances in Computing, Communications and Informatics (ICACCI), pp. 1188-1194. IEEE (2014)

8. Ekman, P., et al.: Universals and cultural differences in the judgments of facial expressions of emotion. J. Pers. Soc. psychol. 53(4), 712 (1987)

9. Enobio: http://www.neuroelectrics.com/products/enobio/

10. Epoc, E.: www.emotiv.com

11. Hamann, S.: Mapping discrete and dimensional emotions onto the brain: controversies and consensus. Trends Cogn. Sci. 16(9), 458-466 (2012)

12. Levenson, R.W.: Basic emotion questions. Emot. Rev. 3(4), 379-386 (2011)

13. Lindquist, K.A., Wager, T.D., Kober, H., Bliss-Moreau, E., Barrett, L.F.: The brain basis of emotion: a meta-analytic review. Behav. Brain Sci. 35(03), 121-143 (2012)

14. Liu, N.H., Chiang, C.Y., Chu, H.C.: Recognizing the degree of human attention using eeg signals from mobile sensors. Sensors 13(8), 10273-10286 (2013)

15. Liu, Y., Sourina, O.: EEG-based subject-dependent emotion recognition algorithm using fractal dimension. In: 2014 IEEE International Conference on Systems, Man, and Cybernetics (SMC). pp. 3166-3171. October 2014. https://doi.org/10.1109/ SMC.2014.6974415 
16. Liu, Y., Sourina, O., Nguyen, M.K.: Real-time EEG-based emotion recognition and its applications. In: Gavrilova, M.L., Tan, C.J.K., Sourin, A., Sourina, O. (eds.) Transactions on Computational Science XII. LNCS, vol. 6670, pp. 256-277. Springer, Heidelberg (2011). https://doi.org/10.1007/978-3-642-22336-5_13

17. Maki, Y., Sano, G., Kobashi, Y., Nakamura, T., Kanoh, M., Yamada, K.: Estimating subjective assessments using a simple biosignal sensor. In: 2012 13th ACIS International Conference on Software Engineering, Artificial Intelligence, Networking and Parallel \& Distributed Computing (SNPD), pp. 325-330. IEEE (2012)

18. Quesada-Tabares, R., Molina-Cantero, A.J., Gómez-González, I., Merino-Monge, M., Castro-García, J.A., Cabrera-Cabrera, R.: Emotions detection based on a single-electrode EEG device. In: Proceedings of the 4th International Conference on Physiological Computing Systems, vol. 1: PhyCS, pp. 89-95. INSTICC, SciTePress (2017). https://doi.org/10.5220/0006476300890095

19. Russell, J.A., Barrett, L.F.: Core affect, prototypical emotional episodes, and other things called emotion: dissecting the elephant. J. Pers. Soc. Psychol. 76(5), 805 (1999)

20. Sanei, S., Chambers, J.: EEG Signal Processing. Wiley, New Jersey (2007)

21. Schafer, R.: What is a savitzky-golay filter? [lecture notes]. Signal Process. Mag., IEEE 28(4), 111-117 (2011). https://doi.org/10.1109/MSP.2011.941097

22. Siamaknejad, H., Loo, C.K., Liew, W.S.: Fractal dimension methods to determine optimum EEG electrode placement for concentration estimation. In: 2014 Joint 7th International Conference on Soft Computing and Intelligent Systems (SCIS), and 15th International Symposium on Advanced Intelligent Systems (ISIS), pp. 952-955. December 2014. https://doi.org/10.1109/SCIS-ISIS.2014.7044757

23. Sourina, O., Liu, Y.: A fractal-based algorithm of emotion recognition from EEG using arousal-valence model. In: BIOSIGNALS, pp. 209-214 (2011)

24. Stone, J.V.: Independent Component Analysis: A Tutorial Introduction. MIT Press, Cambridge (2004)

25. Szibbo, D., Luo, A., Sullivan, T.J.: Removal of blink artifacts in single channel EEG. In: 2012 Annual International Conference of the IEEE Engineering in Medicine and Biology Society. pp. 3511-3514 August 2012. https://doi.org/10. 1109/EMBC.2012.6346723

26. Cervantes-De la Torre, F., González-Trejo, J., Real-Ramírez, C., Hoyos-Reyes, L.: Fractal dimension algorithms and their application to time series associated with natural phenomena. In: Journal of Physics: Conference Series. vol. 475, p. 012002. IOP Publishing (2013)

27. Van Hal, B., Rhodes, S., Dunne, B., Bossemeyer, R.: Low-cost EEG-based sleep detection. In: 2014 36th Annual International Conference of the IEEE Engineering in Medicine and Biology Society (EMBC), pp. 4571-4574. IEEE (2014)

28. Vytal, K., Hamann, S.: Neuroimaging support for discrete neural correlates of basic emotions: a voxel-based meta-analysis. J. Cogn. Neurosci. 22(12), 2864-2885 (2010)

29. Wang, Q., Sourina, O.: Real-time mental arithmetic task recognition from EEG signals. IEEE Trans. Neural Syst. Rehabil. Eng. 21(2), 225-232 (2013). https:// doi.org/10.1109/TNSRE.2012.2236576

30. Yoon, H., Park, S.W., Lee, Y.K., Jang, J.H.: Emotion recognition of serious game players using a simple brain computer interface. In: 2013 International Conference on ICT Convergence (ICTC), pp. 783-786. IEEE (2013) 